\title{
Traduire
}

Revue française de la traduction

\section{Des métaphores dans le langage financier ou du paysage boursier balayé par les vents}

\section{Bernhard Lorenz}

\section{(2) OpenEdition}

\section{Journals}

Édition électronique

URL : http://journals.openedition.org/traduire/960

DOI : $10.4000 /$ traduire.960

ISSN : 2272-9992

\section{Éditeur}

Société française des traducteurs

\section{Édition imprimée}

Date de publication : 1 juin 2008

Pagination : 14-36

ISSN : 0395-773X

\section{Référence électronique}

Bernhard Lorenz, «Des métaphores dans le langage financier ou du paysage boursier balayé par les vents », Traduire [En ligne], 217 | 2008, mis en ligne le 01 juin 2008, consulté le 05 mai 2019. URL: http://journals.openedition.org/traduire/960 ; DOI : 10.4000/traduire.960 


\title{
Des métaphores dans le langage financier ou du paysage boursier balayé par les vents(1)
}

\author{
BERNHARD LORENZ
}

\section{Traducteur financier}

Je fêterai bientôt ma vingt-cinquième année de traduction. Je suis devenu traducteur financier exerçant à titre libéral après une quinzaine d'années de travail salarié dans une grande banque allemande à Paris. Baigné par la force des choses dans cette vie des grands B que sont devenus pour moi bourse, banque, business - il y a vingt ans et avant d'entrer dans la banque, j'étais plutôt spécialisé en agriculture, arts et nucléaire - je me suis souvent demandé pourquoi les questions d'argent semblaient si peu intéresser mes collègues, exception faite des éternelles discussions sur les tarifs de traduction au mot, à la ligne, au caractère, témoignant d'une sensibilité à l'économie.

Je souhaite par cette contribution lever un coin du rideau sur ce monde étrange des MM. Nasdaq et autres Stoxx pour faire entrevoir

(1) Cet article est l'adaptation d'une intervention faite en langue allemande à l'occasion de la treizième rencontre annuelle du Réseau franco-allemand des traducteurs à Bruxelles, le 28 octobre 2006. Conçu initialement pour être présenté à un public de traducteurs, il ne présente pas la scientificité d'un article universitaire - notamment en terme de citation des références utilisées - à laquelle je ne prétends nullement. Je souhaite simplement, par une approche ludique d'encyclopédiste tatillon et brouillon, démontrer que les questions d'argent peuvent avoir un côté poético-attrayant, même pour les non-initiés de la bourse ou du langage financier. Les exemples cités sont empruntés, pour l'allemand, aux résumés hebdomadaires de la presse économique et financière de la Bundesbank (Auszüge aus Presseartikeln : Handelsblatt, Financial Times, Frankfurter Allgemeine Zeitung, Börsenzeitung) et pour le français, à mes propres traductions, notamment des rapports de gestion de fonds d'investissement, ainsi qu'au quotidien Les Échos, aux hebdomadaires Investir et au Journal des finances ainsi qu'au site web de Boursorama et à ses divers forums (www.boursorama.fr). 
que le langage utilisé dans le monde des " costumes-cravates " laisse dans certains cas, et notamment dans le contexte économique et boursier, même très technique, une liberté au traducteur qui, par le recours à une langue métaphorique parfois teintée d'une certaine poésie, lui permet souvent de "prendre son pied".

De l'ombre portée par un système bancaire en berne émerge un rai de lumière dont on ne sait encore sill éclaire la profondeur des ténèbres à venir ou l'étroit chemin menant à la rédemption, fut-il pavé de quelques douloureux sacrifices. Le doute, néanmoins, ne saurait constituer une stratégie, aussi, en aquafortistes patentés, graveronsnous sur le court terme l'image d'une configuration de Reverse Head \& Shoulders " actif " engendrant une phase de pull back susceptible d'occlure la béance, prestement suivie par un rebond à l'issue duquel la cote rejoindrait sa résistance majeure, située en 15.70. Soubaitons alors que l'actualité ne soit point un bain si acide quiau lieu de révéler les sillons de notre esquisse, il ne les creuse jusquà l'os... [Soros86 chat de Boursorama - 21.09.2006]

Lidée de cette contribution m'est venue en traduisant certains textes rédigés par des gérants de fonds d'investissements, sommés de présenter régulièrement aux investisseurs les résultats de leur politique de gestion tout en donnant une description de l'environnement où, d'une part, évoluent les entreprises dans lesquelles ils investissent et où, d'autre part, ils ont dû opérer durant la période considérée. Et je constatais qu'il existait une multitude de termes récurrents pour remplacer les expressions : " hausse ", " baisse ", " augmentation ", « réduction ", etc. Je ne donnerai donc pas de cours ni d'aide à la compréhension des techniques financières. Celles-ci sont de plus en plus compliquées et souvent élaborées par des mathématiciens (certaines banques en emploient aujourd'hui des centaines) pour être ensuite enrobées et rendues publiables ou distribuables par des experts en marketing, ce qui explique que, bien des fois, compte tenu de la segmentation croissante des métiers, certains agents de banque, notamment votre " conseiller personnel ", ne comprennent pas toujours eux-mêmes les instruments développés par les spécialistes mathématiciens. 
Vous échapperez donc à des textes techniques du type suivant, extrait du commentaire d'un graphique publié sur boursorama.com :

L'analyse d'hier, l'apparition du diamant et son scénario baissier CT prend un sacré coup dans le carat! Nouveau rebond, swing point haussier en harami blanc et reprise des volumes. Le titre repart depuis sa MM10 vers le début de la zone de résistance sur 190e.

[...] L'indice est dans une vague haussière depuis le 14 juin. Le début de la hausse s'est fait avec une poussée B, qui a été corrigée totalement par la $C$ suivante. Reprise de la hausse, mais elle devrait être assez lente.

Pour éviter de perdre bon nombre de mes lecteurs, j'ai préféré me concentrer sur des extraits du type suivant, extrait du blogue d'un trader :

À l'aube, une petite bande de taureaux, peu nombreux mais bien organisés, est passée à l'attaque du camp des ours. Ceux-ci, privés de leurs chefs, appelés aussi zinzins, ont tenté de résister jusque vers $14 \mathrm{~h} 30$.

Nouvelle attaque des taureaux provoquant débandade, puis panique et retraite chez les ours. Défaite totale des ours, beaucoup de sang versé, on compte les morts.

Pas de doute, le texte ci-dessus utilise une forte concentration d'images (métaphores) pour décrire une réalité financière, le déroulement d'une journée boursière avec une hausse difficile (due à l'intervention des institutionnels) et une forte baisse qui laisse des investisseurs "sur le carreau ».

J'ajouterai enfin qu'à l'occasion de la structuration, de la classification puis de l'enrichissement de mes idées initiales, j'ai découvert le travail considérable de quelques collègues traducteurs qui ont réfléchi à la question de manière autrement plus sérieuse et je me suis donc, à cette fin, largement appuyé sur leurs travaux, notamment pour la catégorisation des métaphores. Toutefois - et c'est là mon unique " originalité " - je me limite à des exemples allemand - français, tandis que mes chers confrères se sont davantage concentrés sur la paire linguistique incontournable en traduction financière à savoir anglais - allemand(2).

(2) Voir bibliographie. 
Le langage économique et financier à proprement parler a été relativement peu étudié en linguistique. Ce déficit est notamment dû à la complexité du domaine économique ainsi qu'au langage économique lui-même. Nous rencontrons du vocabulaire économique aussi bien dans la langue générale que dans le contexte de spécialités, d'où la difficulté fréquente à les délimiter. Il est plus facile de faire la différence entre le langage de la pharmacie et le langage commun. Des termes " techniques " tels que bourse, banque, acheter/vendre font depuis longtemps déjà partie de la langue générale, tandis que le quidam ne pourra pas toujours dire ce qu'est un algodysfonctionnel de l'articulation temporomandibulaire ni une gouttière occlusale.

La langue économique est l'ensemble de tous les langages spécifiques et de tous les moyens linguistiques utilisés dans un domaine de communication délimité, l'économie, afin de garantir la compréhension de tous les publics concernés. Les notions de langue économique ou de langue de spécialité comprennent divers vocabulaires spécifiques et techniques utilisés par des groupes de personnes ayant des formations, des connaissances et des activités différentes ainsi que des objectifs et formes de communication différents dans un environnement professionnel, universitaire ou scolaire lié, à des degrés divers, aux domaines de l'économie (Buhlmann, 1989).

Les lecteurs ou traducteurs de textes en rapport avec l'économie sont confrontés à des types de documents allant d'articles de la presse quotidienne ou hebdomadaire à des extraits d'ouvrage de spécialité et à des rapports de spécialistes du domaine de l'audit par exemple, en passant par les informations boursières ou les informations économiques diffusées à la télévision et par les commentaires des cours de bourse.

Il s'agit là d'un langage économique et financier d'une grande densité, associé à des noms d'entreprise, des cours, des cotations, des abréviations et enrichi de termes techniques dont le décodage est souvent réservé aux experts. Des termes tels que " rendement de dividende " 
(Dividendenrendite), "capitalisation du marché " (Marktkapitalisierung), Call, Put et IPO (introduction en bourse) exigent du lecteur une connaissance plus ou moins approfondie du métier.

À côté du vocabulaire ou de la terminologie technique spécifique du domaine bancaire ou de la gestion, il faut également citer les quatre caractéristiques suivantes qui déterminent le langage terminologique de l'économie et de la bourse :

- Transfert au second plan de l'agent sémantique, qu'il soit propriétaire du capital ou actionnaire ;

- Personnification des objets, des événements ou des faits abstraits de l'action humaine apparaissant en tant que sujets ;

- Opposition entre densification de l'information et utilisation de métaphores qui la rendent vivante ;

- Usage linguistique stéréotypé avec recours à des formules passepartout (classiques).

Toutes ces caractéristiques font, selon le sociolinguiste V. Arnold (1973), que le gros des lecteurs peine à bien saisir la diversité des informations des pages économiques et financières des quotidiens.

Comme le soulignent A. Tutin et F. Grossmann (2002), selon Heid (1994, 239), il semble y avoir une différence graduelle entre les collocations " conceptuelles", prédominantes en terminologie, et les simples collocations "lexicales", prédominantes dans la langue générale. Pour lui, le sous-langage de la Bourse constituerait un stade intermédiaire entre ces deux extrémités. En fait, plutôt que d'attribuer la différence de comportement des collocatifs à leur appartenance à la "langue générale " ou aux langues de spécialité, on peut se demander si certains domaines de l'expérience, en particulier ceux dans lesquels les référents concrets sont prédominants, ne se prêtent pas plus facilement aux "collocations conceptuelles " que les domaines plus abstraits. Le domaine de la bourse présente en effet de nombreux points communs avec le champ sémantique des émotions. Les noms y sont majoritairement abstraits (hausse, ...) et les métaphores n'y sont pas absentes (bondir, s'effondrer). 
Selon V. Arnold, le langage de la bourse devient aussi une sorte de « langage des dominants»(Herrschaftssprache). Toutefois (et heureusement) le maintien de cette domination ne serait pas le résultat d'une manipulation du contenu mais, tout simplement, de la façon de concevoir et de créer l'information par la langue. En référence à "l'information ", voici une petite liste non exhaustive de dénominations d'un type d'instruments financiers, les certificats, mettant en évidence le jeu constant, dans la dénomination des produits financiers, entre technicité et images. C'est le cas du " certificat airbag ", qui protège contre les baisses de cours, mais aussi de tous ceux énumérés ciaprès :

Turbo-Bear-Scheine, Turbo-Bull-Scheine, PROTECT-Aktienanleihen, Discount-Zertifikate, Endlos-Index-Zertifikate, Sprint-Zertifikate, Hebel-Zertifikate (également : Turbo- oder Knock-out-Zertifikate) Bandbreiten-Zertifikate (également : Korridor-, DoubleChance-, Speed-, Sprint-, Sprinter-, KickStart-Zertifikate), Bonus-Zertifikate Airbag-Zertifikate (également : R-Bag- oder Protector-Zertifikate) Twin-Win-Zertifikat, Outperformance-Zertifikat mit Anpassungsmechanismus(3).

La créativité linguistique des spécialistes du marketing, toujours à l'affût d'occasions de se distinguer de la concurrence, ne recule devant rien lorsqu'il s'agit de qualifier de nouveaux instruments financiers :

(3) Wikipedia//de.wikipedia.org/wiki/Zertifikat. 


\begin{tabular}{|l|l|l|}
\hline & $\begin{array}{l}\text { Anfangsbetrag } \\
\text { (montant initial investi) }\end{array}$ & $\begin{array}{l}\text { Höchstbetrag } \\
\text { (cours maximum/seuil) }\end{array}$ \\
\hline $\begin{array}{l}\text { Deutsche Bank } \\
\text { DoubleChance-Zertifikate }\end{array}$ & Anfangsbetrag & CAP \\
\hline $\begin{array}{l}\text { Sal. Oppenheim } \\
\text { KickStart-Zertifikate }\end{array}$ & Startpreis & Stopppreis \\
\hline $\begin{array}{l}\text { BNP PARIBAS } \\
\text { Sprinter-Zertifikate }\end{array}$ & Basispreis & CAP \\
\hline $\begin{array}{l}\text { Raiffeisen Centrobank } \\
\text { Speed-Zertifikate }\end{array}$ & Startwert & CAP \\
\hline $\begin{array}{l}\text { Bankgesellschaft Berlin, } \\
\text { Speed-Zertifikate }\end{array}$ & Startkurs & Stoppkurs \\
\hline $\begin{array}{l}\text { SG Warrants } \\
\text { Sprint-Zertifikate }\end{array}$ & Basiskurs & Zielkurs \\
\hline
\end{tabular}

Cet exemple montre combien il importe pour le traducteur financier : a) De bien connaître les termes pivots de la gamme des produits propres à chaque établissement, particularité qu’il partage avec le traducteur technique ;

b) De répertorier les termes de remplacement et les synonymes des verbes et adjectifs utilisés pour être en mesure de varier son expression, non seulement à l'intention du lecteur mais aussi pour lui-même. 
Betty Cohen, spécialiste des co-occurrences, a présenté dans divers travaux de nombreuses variantes de termes, adjectifs qualificatifs et verbes, permettant de compléter et de remplacer des noms d'usage courant. Voici, pour illustration, un tableau qui montre comment faire varier les verbes associés au terme "emprunt(4)" :

Emprunt : Un emprunt est une dette résultant de l'octroi de prêts remboursables à terme qui participent à la couverture des besoins de financement durable de l'entreprise.

(4) G. Lakoff et M. Johnson ont travaillé (1980, p. 7) sur le concept métaphorique et sa capacité à structurer nos perceptions et actions ou activités quotidiennes. Prenons le concept de " temps » et la métaphore conceptuelle LE TEMPS C'EST DE L'ARGENT (Time is money), qu'on retrouve sous diverses formes dans le langage courant :

LE TEMPS C'EST DE L'ARGENT

Vous gaspillez mon temps.

Je n'ai pas de temps à perdre.

\section{ZEIT IST GELD}

Sie vergeuden meine Zeit.

Ich habe keine Zeit zu verschenken.

Ich habe keine Zeit zu verlieren.

Le concept abstrait du temps est métaphorisé dans la culture occidentale avec des expressions qui tournent autour du thème de l'argent. Nous en déduisons que le temps, tout comme l'argent, est perçu comme un bien précieux et une ressource rare, fait conforté par l'utilisation réitérée de la métaphore conceptuelle dans notre culture. Le travail est rémunéré selon les heures travaillées, payé en salaire mensuel, les factures de télécommunications sont calculées sur la base d'unités de temps, les entreprises établissent des budgets annuels, le prix d'une chambre d'hôtel est déterminé à la journée ou à la nuitée (Lakoff et Johnson 1980, p. 8).

Le concept métaphorique LE TEMPS C'EST DE L'ARGENT structure donc des faits et activités quotidiennes. Et on agit vraiment comme si le temps était un bien précieux : le temps est quelque chose qu'on peut économiser, donner, investir, perdre ou gaspiller (sparen, verschenken, investieren, verlieren oder verschwenden), ce que O. Jäkel (1997, p. 23 et sq.) formule comme suit : " les expressions métaphoriques... ne doivent pas être considérées à part mais toujours en tant que réalisation linguistique de métaphores conceptuelles ". 


\begin{tabular}{|c|c|c|c|c|}
\hline Emprunt & Noms & $\begin{array}{c}\text { Verbes : } \\
\text { Emprunt } \\
=\text { sujet }\end{array}$ & $\begin{array}{c}\text { Verbes : } \\
\text { Emprunt } \\
\text { = objet }\end{array}$ & Adjectifs \\
\hline Début & émission & & émettre & \\
\hline & lancement & & lancer & \\
\hline Augmentation & accroissement & s'accroître & accroître & considérable \\
\hline & augmentation & augmenter & augmenter & élevé \\
\hline & & monter & & gros \\
\hline & baisse/ & & réduire & petit \\
\hline & diminution & baisser & restreindre & \\
\hline Fin & réduction & diminuer & clore & \\
\hline & & & liquider & \\
\hline & & & rembourser & \\
\hline & & & restituer & \\
\hline
\end{tabular}

Revenons-en aux métaphores. La plupart des analyses réalisées par des linguistes traitent les métaphores comme un phénomène linguistique individuel qui peut se muer en problème de traduction. Selon Dagut (1976), l'aspect principal d'une métaphore est " l'effet choc qu'elle produit sur le lecteur ". Les expressions métaphoriques sont souvent loin de respecter les règles sémantiques d'une langue et sensiblement enracinées dans la culture de la communauté linguistique concernée. Bien entendu, le traducteur s'efforcera, selon Dagut, de restituer ou de maintenir en vie, dans le texte cible, l'effet de choc ressenti, visé ou obtenu dans le texte source. Si cet effet ne peut pas être reproduit en raison de certains facteurs linguistiques ou culturels, comme l'inexistence d'une expérience culturelle ou d'associations possibles, cette métaphore sera considérée comme intraduisible(5).

(5) Voir l'article de Ulrich Heid :

http://elib.uni-stuttgart.de/opus/volltexte/1999/461/pdf/461_1.pdf, page 155. 
Plusieurs choix se présentent alors au traducteur :

1. La reprise à l'identique :

L'expression métaphorique de la langue source peut être rendue par une expression métaphorique équivalente en langue cible. Le sens et l'image linguistique sont conservés.

Ex : L'argent spéculatif afflue vers les actions.

Spekulative Gelder flossen in Aktien.

\section{La substitution :}

L'expression métaphorique du texte source n'a pas d'équivalent direct en langue cible. L'image est rendue par une métaphore de sens comparable et induisant des associations du même ordre.

Ex. : Le marché des actions est malade.

Der Aktienmarkt kränkelt.

\section{La paraphrase:}

La métaphore du texte source est rendue dans le texte cible par une expression non métaphorique.

Ex. : Le marché boursier s'est éteint.

Der Aktienhandel ist zum Erliegen gekommen.

Notons, en anglais : Stock market trading has died.

\section{La compensation :}

Il s'agit de l'inversion du processus de traduction. Une expression non métaphorique du texte source sera rendue par une métaphore dans le texte cible.

Le traducteur a le choix du procédé de traduction, choix qui dépend néanmoins de divers facteurs, dont la fonction du texte cible et celle de la métaphore dans ce texte. Il analysera si la métaphore est habituelle ou usuelle en langue cible et en quoi la métaphorisation contribue à la fonction du texte cible(6), autre facteur déterminant du choix

(6) Voir aussi Walther, $1986: 162$ et sq. et $1990: 443$. 
du procédé de traduction. En règle générale, la métaphore fonctionne comme un instrument permettant l'optimisation de la connaissance, comme un moyen linguistique d'enrichir la langue, et est en rapport avec le type de texte. Dans un texte littéraire, l'auteur peut, par l'utilisation de métaphores, créer de nouvelles approches ou manières de voir, en utilisant pleinement le potentiel créatif des métaphores. Par contre, le scientifique utilise la métaphore comme un "modèle de description et de structuration "(Walther, 1990 : 447) et vise l'exactitude scientifique.

On pourrait étendre le propos aux expressions métaphoriques regroupées ci-après sous forme encyclopédique, par famille métaphorique, et non alphabétique comme dans un dictionnaire, et sans nécessairement proposer ni chercher d'exemples de traduction " équivalente " ou " exacte " entre le français et l'allemand. Les exemples sont catégorisés par champ métaphorique envisageable, de façon à ménager un espace de liberté créatrice dans le choix des traductions. Pour structurer néanmoins ce travail, je me suis fondé sur l'arborescence très détaillée élaborée par Gudrun Sturmann, en me limitant aux métaphores relevant des catégories suivantes : météorologie, liquide, mouvement, alpinisme, santé, conflits.

\section{Météorologie}

\section{1. Climat}

Le climat des affaires en Allemagne s'est légèrement dégradé en février Das Geschäftsklima hat sich wieder abgeschwächt

Une sage décision dans cette météo boursière très particulière Nächste Woche sollte wieder freundlicheres Börsenwetter aufziehen

L'indice VIX de la volatilité - également baptisé baromètre de la peur à Wall Street $-\mathbf{a}$ atteint brièvement son plus haut niveau en près de 24 cinq ans, à 37,50. 
Auf Aussagen von EZB-Präsident Jean-Claude Trichet... reagiert das Börsenbarometer kaum.

L'embellie conjoncturelle de l'année passée

Signe précurseur d'un début d'accalmie

Aufgehelltes Konjunkturbild

Die Wirtschaft läuft auf Hochtouren, kein Wölkchen trübt den Konjunkturhimmel $(\mathrm{CH})$

\subsection{Froid / chaud}

Refroidissement de l'immobilier

Le refroidissement économique américain a pesé sur la tendance

Der Wohnungs- (Immobilien-, Automobil- etc.) markt hat sich abgeküblt

...dass bei Analysten und institutionellen Investoren nach der jüngsten ZEW-Messung geradezu Eiszeit angesagt ist. Der DAX ist erfroren, der Wert ist im Schnee stecken geblieben

Dort, wo besonders viel beiße Luft drin war, hat es am lautesten gescheppert

\subsection{Vent}

La crise du crédit fait souffler un vent de panique !

Panik an Asiens Börsen - Aktienkurse stürzen weiter

Un vent favorable souffle sur les introductions en bourse

Comme vous le constaterez, les vents boursiers ne nous sont pas favorables à cet instant

Börse spürt Aufwind

Ces vents contraires qui soufflent sur Areva.

Die deutsche Börse segelt momentan mit wenig Wind in den Segeln Die Kurse spüren Gegenwind

Les Bourses d'Asie-Pacifique ont subi mercredi un nouveau coup de tabac 


\subsection{Tempête / Orage}

Tempête en bourse ce lundi 21 novembre

Für stürmische Börsenzeiten

Avis de tempête monétaire de l'ADB

Sturmwarnung bei 6.000 Dax-Punkten

Une tornade boursière s'abat sur toutes les places mondiales Les marchés d'actions ont connu une période de fortes turbulences Turbulenzen an den Börsen

Le trou d'air s'est en effet mué en une crise de confiance généralisée Brauchen wir jetzt nicht noch ein wirklich reinigendes Gewitter an der Börse?

Das Sommergewitter an der Börse sei überstanden

\section{Liquide}

Ein bestimmter Teil des Kapitals fließt in Emerging Markets avec la décrue des prix pétroliers Im vergangenen Jahr floss so viel Geld in Schwellenländer wie nie zuvor. Aktienfonds, die in Schwellenländer investieren, verzeichneten Rekordzufliusse von 22,4 Milliarden Dollar

Über Repatriierungsströme scheint davon zumindest kurzfristig sowobl der Dollar profitieren zu können

Si on relie ces éléments aux flux de liquidités déversés sur les marchés les banques occidentales nagent sur un extraordinaire océan de liquidités

Ces liquidités qui ont inondé...

Liquiditätsschwemme

In Zeiten der weltweiten Vernetzung von Kapitalströmen Sorgen machen auch die anhaltend hohen Liquiditätszufliisse. ...gibt es Liquidität im Überfluss - Geld im Überfluss 
"Nous avons des montagnes de liquidités à réinvestir ", explique Alan Wilson

Privatanleger sitzen auf hohen Liquiditätsbergen

La BOE douche les marchés en relevant son repo à 5,25\% Zweite kalte Dusche für SAP

Les banques centrales ont ouvert les vannes de crédit

On ne va pas fermer le robinet du crédit.

frisches Geld in die Märkte gepumpt, um unerwartete Liquiditätsschwankungen rasch ausgleichen

Die Zentralbanken[mussten] den Geldmarkt kurzfristig sogar "fluten ».

\subsection{Sécheresse}

Ce qui se passe sur les marchés boursiers a causé un assèchement des liquidités

La crainte d'un assèchement du crédit alors que la crise du subprime s'étend

Die Austrocknung des Kreditmarktes durch die Subprime-Krise bilden eine Gemengelage

Les liquidités se tarissent sur le marché

Liquiditätsengpässe bringen Finanzbranche ins Wanken

Im Sog des Geldes

die Nachfrage dïmpelt vor sich bin

\section{Mouvement}

\subsection{Direction}

On ne peut exclure un mouvement latéral de grande envergure In der Tat sind die Edelmetalle nach diesem quälenden Seitwärtstrend nun endlich nach oben ausgebrochen

...erkennt auch Flachbewegungen oder Märkte mit Seitwärtsbewegungen 
Bei 10.725 Punkten hat der DOW die Chance einer Gegenbewegung, sollte er jedoch ausschließlich in eine bärische Seitwärtskonsolidierung übergehen

Le titre progresse donc au-delà de l'objectif des 39E prédéterminé par La règle du balancier

Nouvelle poussée des cours du brut ce lundi

Investitionen treiben die Wirtschaft [...] würde der Standard \& Poor's 500 Index [...] bis auf 1100 Punkte vorrücken

Die US-Indizes pendelten zwischen Verlustzone und Gewinnen hin und her auf den Wachstumspfad einschwenken

....a reculé de plus de $\mathrm{x} \%$ de son plus haut niveau historique

Die Aktie ist von einem Höchststand im Juni von 20,36 Euro fast kontinuierlich gesunken

Die Abwärtsbewegung konnte bei 10,725 Punkten erwartungsgemäß gestoppt werden

\subsection{Dynamisme}

Le pétrole s'envole

La formidable envolée qu'a connue la place ces derniers mois.

der Beginn eines Krieges könnte die Börse beflügeln

Erdölpreis macht Sprünge

Les cours bondissent. ING frémit, Fortis et Dexia s'envolent

Franchir la barre des 4300 points

\subsection{Moteur}

Anspringen des Konjunkturmotors...

Les exemples ont montré que le démarrage économique était favorisé par...

Der Konjunkturmotor läuft nun auf zwei von drei Zylindern. Der Konjunkturmotor [...] lief nur mit einem Zylinder (Export) - mit den Investitionen erhielt er zusätzliche Pferdestärken 
Der Konjunkturmotor, der dritte Zylinder stottert gehörig Le moteur conjoncturel suisse n'en finit pas de tousser

Lactivité de fusions-acquisitions, important carburant du marché ces derniers mois

Die Wirtschaft läuft rund.

Strategie deshalb : Nicht "mit Volldampf voraus "

L'économie tourne encore à plein régime en Europe

...après une telle flambée, il est logique que la machine se calme un peu

Voilà que la machine s'emballe

\subsection{Vitesse / lenteur}

Impulse zur Ankurbelung des Konsums.

Le rythme de l'économie mondiale va ralentir

beachtliches Wachstumstempo

L'Afrique a revendiqué une vitesse de croissance la plus rapide de n'importe quelle région du monde

Rohstoffe bleiben aber die Handbremse für die Performance.

Cette évolution reflète l'effet ralentisseur.

En perte de vitesse

\subsection{Pilotage}

Scénario d'atterrissage en douceur sans récession

US-Immobilien: Sanfte Landung statt Kollaps

Harte oder sanfte Landung? - China wird weiterfliegen

Der Aufschwung ist vom Laster gefallen

Il faut mettre le cap sur la croissance et l'emploi. (15.03.2003 Assemblée Générale)

... auf die Phase der Stagnation zusteuern

Alles Gegensteuern des Managements an der Bilanzpressekonferenz nützte nichts 


\section{Alpinisme}

\subsection{Ascension}

auf den Wachstumspfad einschwenken

steile Bergfahrt des Euro

DAX erklimmt neue Höhen

Der Aktienmarkt in New York nimmt eine Rekordmarke nach der anderen, der Gipfel ist aber noch nicht erreicht.

Dass der Dax sich im vergangenen Jahr auf knapp 5500 Punkte hochschleppt

Le prix d'achat de l'immobilier à la montagne a atteint des sommets Neuf Cegetel grimpe entre rumeur et démenti

L'indice a terminé sur un plus haut annuel, retrouvant ses sommets de décembre 2000.

\subsection{Descente}

Wall Street reperd de l'altitude

La Bourse de Paris a poursuivi sa glissade en ouverture

Hatte es zu Wochenbeginn noch nach einer richtigen Talfahrt an den internationalen Börsen ausgesehen

Es geht bergab

Aktienwerte befinden sich noch in einer Talsoble

Höhen und Tiefen der Kursentwicklung

ehe der Abstieg auf ein Tief von 10,50 Euro begann

Laction a dévissé hier de plus de $30 \%$

La descente aux enfers des bourses asiatiques

Vergessen ist das Tal der Tränen

Pendant la dégringolade du CAC 40

La bourse de Paris dégringole sous les 5300 points

Der Kursrutsch zeigt, dass Investitionen in Emerging Markets riskant sind. Zur selben Zeit purzelten auch in Thailand die Aktienkurse.

30 de $3 \%$ 


\section{Schuldenlawine}

...le risque de déclenchement d'une avalanche de pertes et d'une réaction en chaîne

Deutschland fällt wieder auf den flachen Wachstumspfad zurück

\section{Santé}

\subsection{Bonne santé}

La Bourse de Shanghai de nouveau en pleine santé

Weltwirtschaftliche Entwicklung in blendender Verfassung

La semaine fournira encore de précieux éclairages sur la santé économique des États-Unis

Je m'intéresse à cette valeur qui a un bilan sain...

Der Verband nähere sich der 100-Mitglieder-Marke und weise eine gesunde Bilanz und Erfolgsrechnung auf $(\mathrm{CH})$

\subsection{Humeur}

La consommation hésitante

La bourse a affiché une timide hausse

Les marchés financiers retrouvent leur sang-froid

Les investisseurs sont décidément d'humeur changeante

La communauté financière a froidement accueilli le gonflement des stocks

Le coup de blues est maintenant largement perceptible en Europe Auf der anderen Seite kommt neue Lust in den Markt durch das Gerïcht,

Crise boursière : La nervosité s'estompe Investoren werden immer nervöser

Les investisseurs tentaient de résister à la morosité ambiante.

Die Stimmung der Verbraucher hat sich eingetrübt

Séance " funèbre " sur les places européennes 


\subsection{Drogue / Dépendance}

Après dix mois d'abstinence boursière

Aktienabstinenz

L'euphorie s'empare des marchés

Im Zinsrausch

die Konjunktur wird unbotmässig aufgeputscht

Entzug von der Liquiditätsdroge

Die Titel von Barry Callebaut im Höhenrausch

\subsection{Maladie / Fatigue}

La Bourse est malade?

Die Tokioter Börse kränkelt

C'est un effet de contagion

Les Bourses d'Asie-Pacifique contaminées par les places américaines et européennes

Les crises boursières [...] font référence à un effondrement brutal des cours des titres

An den Börsen grassiert immer mehr die Enronitis (affaire Enron)

Der jüngste Kollaps der asiatischen Börse

Un petit essoufflement (sans gravité) de l'activité

L'indice phare de la place s'est accordé une séance pour reprendre son souffle

Dem Dax geht die Luft aus

"Elle est anesthésiée " (l'action ne bouge guère avec très peu de titres échangés)

«Elle a le cul lourd"

Während der Neue Markt [...] ist das heimische Pendant AGM derzeit so gut wie tot

Quand la démocratie actionnariale balbutie, les investisseurs avalent des pilules amères.

Auf diese bitteren Pillen für die Anleger reagierten zahlreiche 
Les perfusions quotidiennes des banques centrales ne règlent rien Liquiditätsspritzen im dreistelligen Milliardenbereich

Le marché du travail a donné des signes de fatigue en juillet Den Kursaufschwung an den Aktienmärkten verschlafen

Fébriles en fin de semaine dernière, les investisseurs ne semblent plus trop préoccupés

\subsection{Alimentation}

" La hausse nourrit la hausse " et « la baisse entraîne la baisse ». Die Hausse nährt die Hausse

Des indices tels que l'indice d'appétit au risque FOMC-Minutes verderben Fondsmanagern den Risikohunger Die Veröffentlichung des Protokolls [...] sowie neuerlich eingetrübte Konjunkturerwartungen haben den Fondsmanagern weltweit den Appetit auf Risiko verdorben (18.1.2005 - Dow Jones)

\section{Conflit / Guerre}

\subsection{Affrontement}

Coventree lutte pour sa survie

Die Prager Börse kämpft [...] ums Überleben

Altadis : vers une bataille boursière de grande envergure ?

Übernahmeschlacht tobt jetzt an der Börse

Pour finir, le " champ de bataille allemand » n'est pas délaissé.

Unser erstes Schlachtfeld sind jetzt die Aktienoptionen

Les hostilités ouvertement lancées dans l'énergie sur le front des prix, ...sur le front des valeurs, General Electric a gagné 3,18 \%...

...mauvaises surprises sur le front macroéconomique...

Rube an der Preisfront. Wie immer, wenn es um die Preise geht

Konflikt [...] eskaliert auch an der Wirtschaftsfront

Die New Yorker Aktienmärkte brachen am Donnerstag auf breiter Front ein 


\subsection{Conquête}

Reconquérir des parts de marché des investisseurs institutionnels Schlussauktion konnten die Vortagesstände aber bis Handelsende wieder zurück erobert werden Zertifikate erobern die Bankschalter

Die deutschen Finanzkonzerne wollen Zertifikate mit einer offensiven Vertriebs- und Marketingstrategie als Massenprodukt etablieren

La stratégie publicitaire va se concevoir en fonction des marchés visés die angepeilten Ertragsziele verfehlen werde

\subsection{Armes}

Feux nourris d'avis d'analystes

Les investisseurs potentiels sont pris sous les feux croisés d'un consensus très convaincant

Nach dem Einbruch am US-Immobilienmarkt geraten nun die Ratingagenturen unter Beschuss.

Dans le monde ancien, les armes monétaires et budgétaires permettaient aux états de provoquer la demande

Geldpolitische Schwerter sind stumpf

Guerre monétaire (non conventionnelle)

\subsection{Fuite}

Les investisseurs ont fui les titres à haut rendement An den Rohstoffmärkten ergriffen die Investoren ebenfalls die Flucht. 


\section{Bibliographie}

Arnold Volker (1973) : "Kritische Analyse des Sprachgebrauchs der Wirtschaftsjournalistik in Tageszeitungen, in J. B. Metzler : Projekt Deutschunterricht 4. Stuttgart, p. 94-119.

Buhlmann Rosemarie (1989) : "Fachsprache Wirtschaft gibt es die? " JBDaF 15, 1989.

Cohen Betty (1986) : Lexique de Co-occurrents. Bourse-Conjoncture Économique. Montréal, Linguatech.

Cohen Betty (1992) : "Méthodes de repérage et de classement des co-occurrents lexicaux ", Terminologie et traduction. Vol. 2-3 (1992), Cahier 3, p. 505-511 [BL 2].

Dagut Menachem B. (1976) : "Can ,metaphor' be translated ", in : Babel xxii/1, p. 21-33.

Jäkel Olaf (1997) : Metaphern in abstrakten Diskurs-Domänen. (Duisburger Arbeiten) zur Sprach- und Kulturwissenschaft 30), Frankfurt, Wien etc., Lang.

Krenkova Dagmar (2003) : Phraseologismen in der Deutschen Sportpublizistik - Inaugural-Dissertation zur Erlangung des Doktorgrades - Masaryk-Universität-Brno (28.5.2003)

Lakoff George (1987) : Women, Fire and Dangerous Things. Chicago, London, The University of Chicago Press.

Lakoff George (1992) : "Metaphor and war : The metaphor system used to justify war in the Gulf ", in : Pütz, Martin (éd.) Thirty Years of Linguistic Evolution, Benjamins, Philadelphia, Amsterdam, p. 463481 .

Lakoff George/Johnson, Mark (1980) : Metaphors we live by, Chicago, London, The University of Chicago Press.

Lakoff George/Turner, Mark (1989) : More than Cool Reason - A Field Guide to Poetic Metaphor, Chicago, The University of Chicago Press. 
Osthus Dietmar (2000) : Metaphern im Sprachvergleich. Eine kontrastive Studie zur Nahrungsmetaphorik im Französischen und Deutschen. (Bonner romanistische Arbeiten 70), Frankfurt, Wien, Lang.

Sturmann Gudrun (2005) : Die Wiener Börse - Die Terminologie im Deutschen und im Englischen -Diplomarbeit zur Erlangung des akademischen Grades einer Magistra der Philosophie an der Geisteswissenschaftlichen Fakultät der Karl-Franzens-Universität Graz (Institut für Theoretische und Angewandte Translationswissenschaft) [La première partie est consacrée à la Bourse de Vienne (structures et terminologie anglais - allemand), la deuxième traite des métaphores utilisées dans le langage boursier (anglais - allemand).]

Tutin Agnès, Grossmann Francis (2002) : "Collocations régulières et irrégulières : esquisse de typologie du phénomène collocatif, Revue française de Linguistique Appliquée, Lexique : recherches actuelles, vol. VII, p. 7-25.

Walther Wolfgang (1986) : «Neue Aspekte der Übersetzung von Metaphern in journalistischen Texten (E-D) », in : Fremdsprachen 30, p. 162-166, p. 237-242.

Walther Wolfgang (1990) : «Faktoren für die Übersetzung von Metaphern (Englisch-Deutsch) », in Arntz, Reiner/Thome, Gisela (Hrsg.) Festschrift für Wolfram Wilss zum 65. Geburtstag. Übersetzungswissenschaft. Ergebnisse und Perspektiven. Tübingen, Narr, p. 440-452.

Liens en rapport avec les métaphores

http://www.metaphorik.de/Journal/

http://www.metaphorik.de/03/amoraritei.htm (Métaphores dans le vin) http://www.metaphorik.de/02/michels.htm (Métaphores dans les reportages de foot en France)

http://www.metaphorik.de/05/rezensionbeissner.htm (Métaphores dans le domaine de la nourriture)

http://www.metaphorik.de/aufsaetze/jamet-internet.htm (Denis Jamet : Comment surfer sur les autoroutes de l'information sans se prendre les pieds dans la corbeille) 